\title{
Small duodenal gastrointestinal stromal tumor presenting with acute bleeding misdiagnosed as hemobilia: Two case reports
}

\author{
CHANGWEI LIN $^{1 *}$, YETING CHANG $^{2 *}$, YI ZHANG $^{1}$, YUNFEI ZUO $^{3}$ and SHUANGYI REN ${ }^{1}$ \\ Departments of ${ }^{1}$ General Surgery and ${ }^{2}$ Cardiovascular Medicine, The Second Affiliated Hospital of Dalian Medical University, \\ Dalian 116023; ${ }^{3}$ Department of Clinical Biochemistry, Dalian Medical University, Dalian 116044, P.R. China
}

Received April 2, 2012; Accepted July 11, 2012

DOI: $10.3892 / \mathrm{ol} .2012 .838$

\begin{abstract}
Only 3-5\% of gastrointestinal stromal tumors (GISTs) are located in the duodenum. They are associated with an increased risk of fatal gastrointestinal bleeding, which is a primary manifestation. A small GIST (less than $2 \mathrm{~cm}$ in size) is easily confused with a duodenal papilla. In the 2 cases presented in this study, endoscopic examination alone resulted in a misdiagnoses of hemobilia. To the best of our knowledge, this is the first case report of this type in the literature. Patient 1 is a 66-year-old male who was admitted to The Second Affiliated Hospital of Dalian Medical University with syncope. Emergent upper gastrointestinal endoscopy identified bleeding from what appeared to be a duodenal papilla, and the patient was diagnosed with hemobilia. However, the angiography did not support this result. To save the patient's life, an exploratory laparotomy was conducted. We identified a small tumor in the descending part of the duodenum and a wedge resection was successfully conducted. The final diagnosis was duodenal GIST with no further risk to the patient. Patient 2 is a 71-year-old female who was admitted to the hospital diagnosed with hemobilia. The patient underwent a barium swallow examination and a contrast-enhanced computed tomography (CT) scan, in which a tumor on the descending part of the duodenum was identified. Patient 2 also underwent a wedge resection. The final diagnosis was duodenal GIST with no further risk to the patient. Gastroscopy may be a useful procedure for duodenal GIST diagnosis; however, the
\end{abstract}

Correspondence to: Professor Shuangyi Ren, Department of General Surgery, The Second Affiliated Hospital of Dalian Medical University, 467 Zhongshan Road, Shahekou, Dalian 116023, P.R. China

Email: rsydl@yahoo.com.cn

Professor Yunfei Zuo, Department of Clinical Biochemistry, Dalian Medical University, 9 West Section, Lvshun South Road, Dalian 116044, P.R. China

E-mail: zyf04112002@yahoo.com.cn

*Contributed equally

Key words: small duodenal gastrointestinal stromal tumor, misdiagnosed, hemobilia endoscopic findings may be confused with hemobilia when the tumor diameter is less than $2 \mathrm{~cm}$ in size and heavily accompanied with blood clots. A barium swallow examination and contrast-enhanced CT may prevent a misdiagnosis of hemobilia.

\section{Introduction}

Gastrointestinal stromal tumors (GISTs) are the most common type of mesenchymal tumor of the gastrointestinal tract (1). The majority of GISTs test positive for mutations in the v-kit Hardy-Zuckerman 4 feline sarcoma viral oncogene homolog (KIT) gene. This leads to the expression of CD34 or the protein marker CD117, which is also known as the mast/stem cell growth factor receptor (SCFR) and c-Kit (2). Approximately 60,30 and $1-2 \%$ of GIST cases occur in the stomach, small intestine and the colon or rectum, respectively. GISTs of the esophagus are rare (3).

The most common presentation of GIST is bleeding of the upper gastrointestinal tract, which may be either acute or chronic, and results in anemia (4). Complete surgical resection remains the best treatment option. Unlike carcinomas, GIST does not widely infiltrate at the microscopic level and rarely metastasizes to the lymph nodes; therefore, local excision may be appropriate when technically feasible.

GISTs of the duodenum occur in less than $4 \%$ of all cases and frequently involve the descending portion (5). As the GIST grows, the central portion becomes necrotic and forms a deeply penetrating ulcer, which may result in hematemesis. Under the gastroscope, the tumor may appear similar to a papilla; therefore, it is difficult to distinguish between a patient with hemobilia and a patient with a small duodenal GIST. In the present study, we report 2 cases of small duodenal GIST (less than $2 \mathrm{~cm}$ in size), which at first were misdiagnosed as hemobilia. The study was approved by the ethics committee of the Second Affiliated Hospital of Dalian Medical University. Patient consent was obtained both from the patient and the patient's families.

\section{Case report}

Patient 1. A 66-year-old male was admitted to The Second Affiliated Hospital of Dalian Medical University (Dalian, China) with syncope. The patient presented with heartburn and 


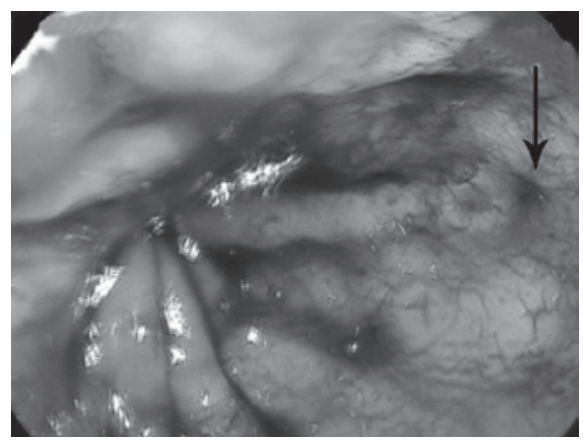

Figure 1. Patient 1. Upper gastrointestinal endoscopic examination revealed an ulcer, heavily accompanied by blood clots, which appeared to be a duodenal papilla.
A

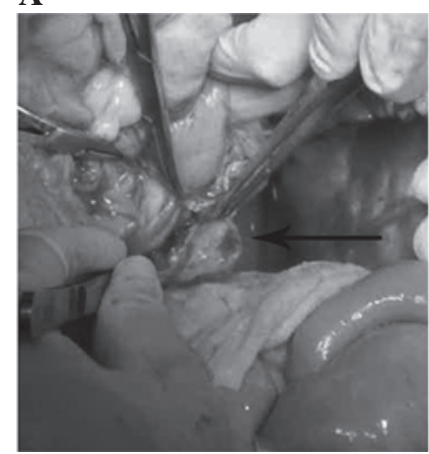

B

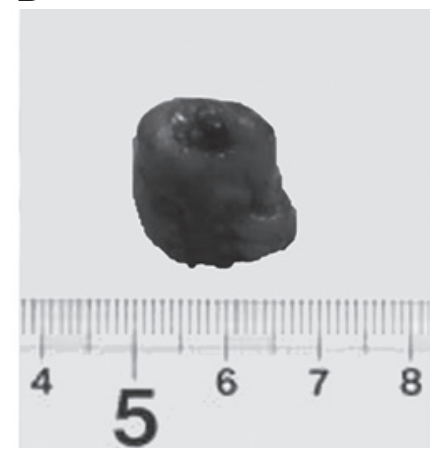

Figure 2. Patient 1. (A) The descending section of the duodenum was incised and an oozing submucosal tumor $(<2 \mathrm{~cm})$ was identified. (B) The tumor measured $1.8 \mathrm{~cm}$ at its greatest dimension and formed a deep ulcer.

melena for 17 days. There was no associated fever, dyspeptic symptoms or localized abdominal pain and the hemoglobin level upon admission was $29 \mathrm{~g} / \mathrm{l}$. Due to the patient's poor condition, a blood transfusion and a high ligation of the great saphenous vein were conducted.

Emergent upper gastrointestinal endoscopy revealed bleeding from a duodenal papilla (Fig. 1), which was diagnosed as hemobilia; however, further angiography did not support this result. Although the blood transfusion level was increased to $12,000 \mathrm{ml}$, the hemoglobin level remained at $41 \mathrm{~g} / 1$ (reference range, $120-160 \mathrm{~g} / \mathrm{l}$ ). There was no time to conduct other accessory examinations. To save the patient's life, an exploratory laparotomy was conducted. During the surgery, numerous blood clots were identified in the second part of the duodenum, and no bleeding was identified in the duodenal papilla. A submucosal tumor $(<2 \mathrm{~cm})$ with signs of ulceration and oozing at the upper end was revealed on the descending section of the duodenum (Fig. 2A and B). The tumor was $<2 \mathrm{~cm}$ in diameter, and $1 \mathrm{~cm}$ from the papilla. Peritoneal dissemination and liver metastasis were not observed.

On the basis of these observations, and since the patient was elderly and weakening, a wedge resection was conducted. The histopathological examination of the resected lesion revealed a GIST. The patient did not undergo chemotherapy following surgery and, to date, has been free from recurrence.
A

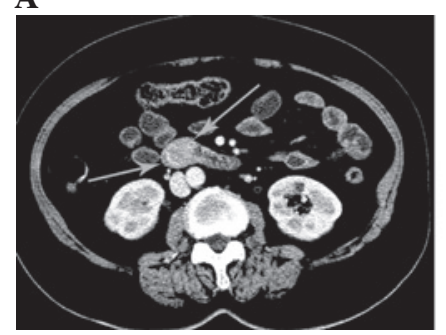

B

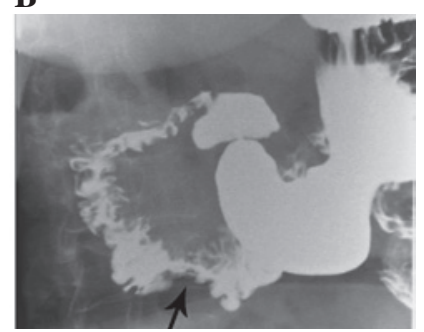

Figure 3. Patient 2. (A) Tumor located in the descending duodenum with typical strengthening CT appearance (arrow, duodenal GIST). (B) Gastrointestinal imaging identifed a circular notch convex to the intestine on the descending duodenum. A and B both indicate the presence of a tumor on the descending duodenum. CT, computed tomography.

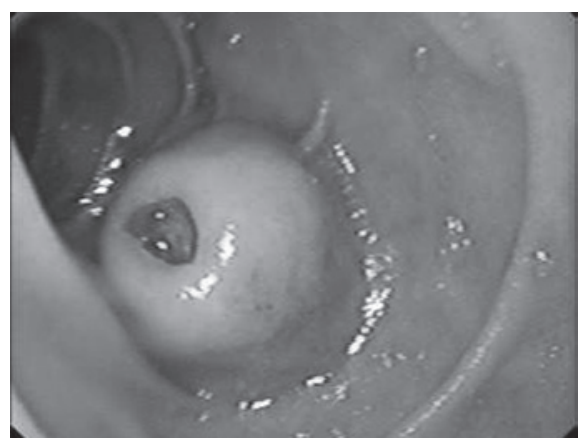

Figure 4. Patient 2. Endoscopic examination revealed a tumor which necrotized in the center and formed a duodenal papilla-like ulcer.

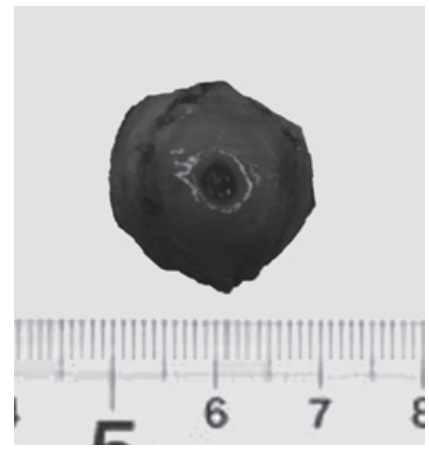

Figure 5. Patient 2. The tumor measured $2.0 \mathrm{~cm}$ at its greatest dimension, with a deep ulceration and necrotic center.

Patient 2. A 71-year-old female with no previous medical history presented with a 2-month history of melena. The patient felt nauseated, suddenly vomited $\sim 50 \mathrm{ml}$ of blood and was immediately taken to a local hospital.

An upper gastrointestinal endoscopy resulted in a diagnosis of hemobilia. The patient was then transferred to The Second Affiliated Hospital of Dalian Medical University for further treatment. From our previous experience with patient 1, we understood that endoscopic diagnosis comprises certain limitations. Therefore, the patient underwent barium swallow and a contrast-enhanced computed tomography (CT) scan, which 
revealed a high-density area in the descending duodenum (Fig. 3A and B).

An endoscopy was then conducted by an experienced practitioner, who identified a tumor $<2 \mathrm{~cm}$ in diameter following sufficient washing of the bleeding site. The tumor revealed an area of necrosis and ulceration, which had a similar appearance to a duodenal papilla (Fig. 4). We temporarily controlled the bleeding by sclerotherapy, then a wedge resection was conducted to permanently terminate the bleeding. During the procedure, we identified that the tumor was notably small and located $\sim 1 \mathrm{~cm}$ from the papilla. Peritoneal dissemination and liver metastasis were not observed.

Gross examination revealed a $1.5 \times 2.0-\mathrm{cm}$ submucosal tumor covered with blood and containing a central ulceration (Fig. 5). Upon histopathological examination, the resected lesion was identified as a GIST. The patient did not undergo chemotherapy following surgery, and to date, has been free from recurrence.

\section{Discussion}

GIST is the most common type of mesenchymal tumor, which appears to originate from the gastrointestinal wall, mesentery, omentum or retroperitoneum. GISTs usually occur on the stomach or small bowel, and only $1-4 \%$ are located in the duodenum. Depending on their size, location and the presence of mucosal ulceration, GISTs most often present with gastrointestinal bleeding, abdominal pain and obstructive jaundice (6).

The most typical feature of a duodenal GIST is the formation of a deep ulceration in the mucosa or an intramural mass with a centrally ulcerated umbilication (7). Therefore, bleeding is the most common symptom at presentation. Bleeding may cause acute abdominal pain and severe anemia, which may require emergency surgery. In the presented cases, the most common symptom was anemia, and in previous studies, anemia and gastrointestinal bleeding were observed in $48 \%$ of cases with ulcerated lesions (8).

The gross ulceration of a GIST in the duodenum facilitates the detection of the tumor under endoscopic examination; however, a small duodenal tumor (less than $2 \mathrm{~cm}$ in size) is easily confused with a duodenal papilla. In the presented cases, the endoscopic diagnosis for both patients was hemobilia. In hemobilia, acute upper gastrointestinal bleeding is one of the most common life-threatening conditions; therefore, the sooner the cause of bleeding is identified, the sooner the patients are out of danger. For this reason, a complete examination should be conducted during upper gastrointestinal bleeding to prevent misdiagnosis.

To date, there are no recognized specific radiological examinations for duodenal GIST diagnosis; however, a gastroscopy may be a useful procedure. Under the endoscope, it is possible to observe the hemorrhagic area, arrest the bleeding and conduct a biopsy. More attention should be paid to the bleeding from the descending part of the duodenum. When there is deep ulceration of the tumor, the endoscopic appearance may be confused with hemobilia by an insuf- ficiently observant gastroscopy operator, particularly when the tumor diameter is less than $2 \mathrm{~cm}$ and markedly accompanied with blood clots. The barium swallow examination may be complementary to gastroscopy in the differential diagnosis of hemobilia and tumor bleeding.

Contrast-enhanced CT is the standard preoperative imaging technique. It usually exposes a mass originating from the digestive tract wall and reveals the tumor size and presence of secondary localizations (e.g., hepatic metastases). In patient 2, the barium swallow examination demonstrated a filling defect, indicating the presence of a mass causing a mucosal irregularity. A contrast-enhanced CT scan revealed a high density lesion in the descending part of the duodenum. Utilizing the barium swallow examination and contrast-enhanced CT scan may help prevent a misdiagnosis of hemobilia. Thus, a gastroscopy, contrast-enhanced CT scan and barium swallow examination are recommended to increase the preoperative diagnostic rate for small duodenal GISTs.

In conclusion, the two duodenal GIST (less than $2 \mathrm{~cm}$ in size) cases presented were initially misdiagnosed as hemobilia. To the best of our knowledge, this is the first published acknowledgment that such a misdiagnosis may be likely. To avoid misdiagnosis, a gastroscopy, contrast-enhanced CT scan and barium swallow examination should be routinely conducted.

\section{Acknowledgements}

This study was supported by grants from the Chinese National Natural Science Foundation Projects (30870550) and the Chinese State Key Program in Basic Research (2012CB822103).

\section{References}

1. Judson I and Demetri G: Advances in the treatment of gastrointestinal stromal tumours. Ann Oncol 18 (Suppl 10): x20-x24, 2007.

2. Yoshida H, Mamada Y, Taniai N, Mizuguchi Y, Nakamura Y, Nomura T, Okuda T, Uchida E, Fukuda Y, Watanabe M and Tajiri T: Spurt bleeding from a calcificated gastrointestinal stromal tumor in the stomach. J Nihon Med Sch 72: 304-307, 2005.

3. Stamatakos M, Douzinas E, Stefanaki C, Safioleas P, Polyzou E, Levidou G and Safioleas M: Gastrointestinal stromal tumor. World J Surg Oncol 7: 61, 2009.

4. Miettinen $\mathrm{M}$ and Lasota $\mathrm{J}$ : Gastrointestinal stromal tumors: review on morphology, molecular pathology, prognosis, and differential diagnosis. Arch Pathol Lab Med 130: 1466-1478, 2009.

5. Chung JC, Chu CW, Cho GS, Shin EJ, Lim CW, Kim HC and Song OP: Management and outcome of gastrointestinal stromal tumors of the duodenum. J Gastrointest Surg 14: 880-883, 2010.

6. Mennigen R, Wolters HH, Schulte B and Pelster FW: Segmental resection of the duodenum for gastrointestinal stromal tumour (GIST). World J Surg Oncol 6: 105, 2008.

7. Gervaz P, Huber O and Morel P: Surgical management of gastrointestinal stromal tumours. Br J Surg 96: 567-578, 2009.

8. Novitsky YW, Kercher KW, Sing RF and Heniford BT: Long-term outcomes of laparoscopic resection of gastric gastrointestinal stromal tumors. Ann Surg 243: 738-744, 2006. 